\title{
Soluble IL-1 receptor 2 is associated with left ventricular remodelling in patients with ST-elevation myocardial infarction
}

Running title: Soluble IL-1R2 in STEMI

Hilde L. Orrem ${ }^{1,2,3}$, Christian Shetelig ${ }^{3,4,5}$, Thor Ueland ${ }^{3,6,7}$, Shanmuganathan Limalanathan $^{4,5,8}$, Per H. Nilsson ${ }^{1,9,10}$, Trygve Husebye ${ }^{4,11}$, Pål Aukrust ${ }^{3,6,9,12}$, Ingebjørg Seljeflot $^{3,4,5,11}$, Pavel Hoffmann ${ }^{3,13}$, Jan Eritsland ${ }^{4,5}$, Tom E. Mollnes ${ }^{1,7,9,14,15}$, Geir Øystein Andersen $^{4,5,11}$, Arne Yndestad $3,6,9,11^{*}$

${ }^{1}$ Department of Immunology, ${ }^{2}$ Department of Anesthesiology, Oslo University Hospital Rikshospitalet, ${ }^{3}$ Institute of Clinical Medicine, University of Oslo, ${ }^{4}$ Department of Cardiology, ${ }^{5}$ Center for Clinical Heart Research, Oslo University Hospital Ullevål, ${ }^{6}$ Research Institute of Internal Medicine, Oslo University Hospital Rikshospitalet, ${ }^{7}$ K.G. Jebsen TREC, University of Troms $\emptyset$, Troms $\varnothing,{ }^{8}$ Feiring Heart Clinic, Feiring, ${ }^{9}$ K.G. Jebsen Inflammatory Research Centre, University of Oslo, ${ }^{10}$ Linnaeus Centre for Biomaterials Chemistry, Linnaeus University, Kalmar, Sweden, ${ }^{11}$ Center of Heart Failure Research, University of Oslo, ${ }^{12}$ Section of Clinical Immunology and Infectious Diseases, Oslo University Hospital Rikshospitalet, ${ }^{13}$ Section of Interventional Cardiology, Oslo University Hospital Ullevål, ${ }^{14}$ Research Laboratory, Nordland Hospital, Bodø, ${ }^{15}$ Centre of Molecular Inflammation Research, Norwegian University of Science, Trondheim, Norway. 


\begin{abstract}
Background. The inflammatory response following myocardial infarction (MI) is prerequisite for proper healing of infarcted tissue, but can also have detrimental effects on cardiac function. Interleukin (IL)-1 $\alpha$ and IL-1 $\beta$ are potent inflammatory mediators and their bioactivity is tightly regulated by IL-1 receptor antagonist (IL-1ra) and soluble (s) IL-1 receptors $(\mathrm{R})$.
\end{abstract}

Objectives. We aimed to examine whether levels of soluble regulators of IL-1 signalling are changed during ST-elevation MI (STEMI) and their associations with parameters of cardiac injury and ventricular remodelling.

Methods. Plasma levels of IL-1Ra, sIL-1R1, sIL-1R2 and sIL-1R accessory protein (sIL1RAcP) were measured by immunoassays in repeated samples from patients with STEMI $(n=272)$ and compared to healthy controls $(n=65)$.

Results. IL-1Ra, sIL-1R1 and sIL-1R2 levels were all significantly elevated after STEMI, while levels of sIL-1RAcP were lower compared to controls. sIL-1R2 levels (at different time points) correlated positively with C-reactive protein, myocardial infarct size and change in indexed left ventricular end-diastolic and end-systolic volume (LVEDVi and LVESVi) measured by cardiac MR acutely and after 4 months, and negatively with LV ejection fraction. Patients with >median levels of sIL-1R2 in the acute phase were more likely to have increased change in LVEDVi and LVESVi. Importantly, sIL-1R2 remained significantly associated with change in LVEDVi and LVESVi also after adjustment for clinical covariates. Conclusion. Levels of sIL-1R2 are independently associated with parameters of LV adverse remodelling following STEMI.

Keywords. interleukin-1, soluble receptors, myocardial infarction, STEMI, ventricular remodelling 


\section{Introduction}

Inflammation has dual role in cardiovascular disease. It is suggested to play a pivotal role in all stages of coronary artery disease from development of atherosclerotic plaques to plaque rupture and thrombus formation, but is also involved in cardiac repair and remodelling following myocardial infarction (MI) [1, 2]. The inflammatory response following MI involves cytokine release and infiltration of neutrophils and monocytes, which is prerequisite for proper healing of damaged cardiac tissue. However, excessive inflammation may cause collateral damage and have detrimental effects on cardiac function both in the short and long term $[1,2]$. Clinical trials have found that targeting interleukin (IL)-1 in acute coronary syndromes by recombinant IL-1 receptor antagonist (IL-1Ra) has anti-inflammatory effects accompanied by reduced ventricular remodelling $[3,4]$, and neutralization of IL-1 $\beta$ was very recently shown to induce a lower rate of recurrent cardiovascular events in patients with previous MI [5]. However, the regulation of IL-1 related molecules, in the complex IL-1 system, following MI are still not clear.

IL-1 $\alpha$ and IL-1 $\beta$ are potent upstream mediators of inflammation and their activity is regulated at several levels (Figure 1) [6]. IL-1 $\alpha$ is often classified as an alarmin and is typically membrane associated and rarely detectable in circulation [6]. Activation and secretion of IL$1 \beta$ is a two-step process involving inflammasomes [6]. IL-1 (both $\alpha$ and $\beta$ ) binds the type I IL-1 receptor (IL-1R1) resulting in recruitment of the co-receptor IL-1 receptor accessory protein (IL-1RAcP), which is required for signal transduction (Figure 1A). To avoid an excessive inflammatory response, IL-1 activity is tightly regulated at the receptor level [6]. IL-1 receptor antagonist (IL-1Ra) competes with IL-1 for binding to IL-1R1 (Figure 1B). Moreover, type II IL-1 receptor (IL-1R2) lacks the ability to initiate signalling and acts as a decoy receptor (Figure 1C). Finally, the extracellular domain of all IL-1 receptors may be 
shed from the cell surface and act as soluble (s) negative regulators of IL-1 signalling (i.e., sIL-1R1, sIL-1R2 and sIL-1RAcP; Figure 1D-G). sIL-1R2 may be of particular importance since binding to IL-1 is nearly irreversible [6], whereas sIL-1R1 also binds IL-1Ra and may therefore also have an inflammatory effect.

We have previously shown elevated levels of IL-1 $\beta$ during acute ST segment elevation MI (STEMI) and an association with subsequent left ventricular (LV) hypertrophy and remodelling [7]. Others and we have also shown that IL-1Ra is increased in the acute phase of MI [7-9], but not much is known about levels of the soluble IL-1 related receptors which clearly influence the net activity in IL-1 related pathways. Herein, we examined levels of soluble regulators of IL-1 signalling in STEMI as well as their associations with parameters of cardiac injury and ventricular remodelling.

\section{Material and methods}

\section{Study population}

Samples from the previously reported Post-conditioning in ST-Elevation Myocardial Infarction (POSTEMI; www.clinicaltrail.gov; NCT00922675) trial [10] were investigated and consisted of 272 patients with first-time STEMI with symptoms of $<6$ hours and with typical changes in ECG with $>1 \mathrm{~mm}$ elevation in the ST-segment in at least two contiguous extremity leads or $>2 \mathrm{~mm}$ elevation of the ST-segment in at least two contiguous precordial leads or new-onset left-bundle branch block and with successful opening of the occluded coronary vessel after one balloon inflation. Patients were randomized to standard reperfusion or to four repeated occlusions of the coronary vessel of one minute duration starting one minute after the first opening of the artery. Patients with previous MI or with renal failure (serum 
creatinine $>200 \mu \mathrm{mol} / \mathrm{L}$ ), unstable patients with cardiogenic shock, pulmonary congestion, hypotension or cardiac arrest were not included in the study. Sixty-five healthy age and sexmatched individuals were included as controls.

The study was approved by the Regional Ethics committee and conducted in accordance with the principles of the declaration of Helsinki and all patients provided written informed consent.

\section{Cardiac magnetic resonance imaging (CMR)}

CMR was performed in the acute phase (median 2 days following MI) and after four months and has previously been described in detail [10]. Briefly, CMR was performed on a 1.5-T scanner (Philips Intera, release 11 or Philips Achieva, release 3.2, Philips Healthcare, Best Netherlands). The patients were examined in the same scanner at both occasions. Image analyses were performed on an extended MR workspace (Philip Medical Systems). LV volume analyses were performed on short axis images. Analysis of area at risk, defined as myocardium with signal intensity of more than two standard deviations above the signal intensity in non-infarcted remote myocardium, was performed on $\mathrm{T} 2$ weighted images in the short axis plane. Infarct size was determined in two- and four-chamber, long and short axis views 15 minutes following contrast injection (Gadolinium-DTPA $469 \mathrm{mg} / \mathrm{ml}, 0.15 \mathrm{mmol} / \mathrm{kg}$, Magnevist, Schering AG, Germany). Myocardial salvage index (\%) was calculated as follow: (area at risk- infarct size at 4 months/ area at risk) x 100. Microvascular obstruction (MVO) was defined as dark areas within the infarcted myocardium.

\section{Blood sampling protocol}

Blood samples were drawn before and immediately after the percutaneous coronary intervention (PCI) procedure, at day 1 following the procedure and after 4 and 12 months. EDTA-blood was placed on crushed ice and within 30 minutes centrifuged at $3000 \mathrm{~g}$ for 20 minutes at $4{ }^{\circ} \mathrm{C}$ to obtain platelet-.poor plasma. Samples were stored at $-80^{\circ} \mathrm{C}$ until analysis. 


\section{Immunoassays}

EDTA-plasma levels of sIL-1R1, sIL-1R2, sIL-1RAcP were analysed by enzyme immunoassays from R\&D Systems (DuoSet), while IL-1Ra was measured using an enzyme immunoassay from Peprotech (Rocky Hill, NJ).

\section{Statistical analyses}

Differences between groups were analysed with the use of Mann-Whitney $U$ tests. When comparing $\geq 3$ groups, Kruskal-Wallis test with Dunn's multiple comparison test was used. Univariate repeated measures ANOVA was used to assess changes in protein levels over time during STEMI. Associations between variables were assessed by means of Spearman correlation coefficient. To further assess possible associations between sIL-1R2 and LV remodelling, multiple linear regression analyses were performed with change in indexed LV end-diastolic volume (LVEDVi) and LV end-systolic volume (LVESVi) from STEMI to 4month follow-up as outcome variables, respectively. The following covariates were entered into the models based on either clinical relevance or an association with either sIL-1R2 or the dependent variable in univariable analyses with a p-value $<0.2$ : Age, gender, hypertension, diabetes mellitus, time from symptom onset to PCI, infarct localization (anterior MI vs inferior or posterior MI), treatment with ischemic postconditioning, peak troponin T, peak Creactive protein (CRP), N-terminal brain natriuretic peptide (NT-proBNP) on admission and haemoglobin on admission. As a result of skewness, the following continuous variables were logarithmically transformed with the natural logarithm (ln): Time from symptom onset to PCI, troponin T, CRP, and NT-proBNP. Pairwise deletion was used to handle missing data in multivariable analyses. Collinearity was assessed by the variance inflation factor in all multivariable models. A p-value $<0.05$ was considered statistically significant. All analyses were performed by IBM SPSS Software, version 23.0 for Windows (SPSS Inc., Chicago, IL). 


\section{Results}

\section{Temporal changes in plasma levels of IL-1 signalling regulators after STEMI}

We analysed plasma levels of regulators of IL-1 signalling in 272 patients with STEMI from the POSTEMI trial [10], and 65 healthy controls (age 66 [25, 75 percentile: 46, 68]; 52\% men). Baseline characteristics are shown in Table 1. Samples were acquired immediately before and immediately after end of the PCI procedure, at day 1 after PCI (median 18.3 hours after PCI) and at 4-month and 12-month follow-up. At baseline, before PCI, the STEMI population was characterized by increased levels of IL-1Ra, sIL-1R1 and sIL-1R2 and decreased levels of sIL-1RAcP as compared with healthy controls (Figure 2). IL-1Ra levels peaked right after PCI, but remained elevated even one year after STEMI. sIL-1R1 levels were at their highest before PCI and thereafter decreased, ending up lower than controls at 4 and 12-month follow-up. Notably, however, the elevated levels of sIL-1R2 and decreased sIL1RAcP levels remained stable throughout the study. The ischemic post-conditioning procedure did not affect levels of IL-1 regulators (data not shown).

\section{Levels of soluble IL-1 modulators in relation to measures of myocardial injury and remodelling as assessed by CMR after STEMI}

We then studied the associations between soluble regulators of IL-1 signalling and markers of myocardial damage and remodelling. There was no association between levels of IL-1Ra and sIL-1R1 and CMR data during the acute phase or CMR data after 4 months (Table 2, Supplementary Table S1 and S2). In contrast, sIL-1RAcP and in particular sIL-1R2 showed some interesting associations (Table 2, Supplementary Table S3 and S4). Whereas sIL-1RAcP was inversely correlated with infarct size measured acutely and at 4 months' follow-up, sIL1R2 measured in the acute phase was associated with LV ejection fraction (LVEF) and infarct 
size measured acutely (negative and positive correlation, respectively) and change in indexed LV end-diastolic volume (LVEDVi) and LV end-systolic volume (LVESVi) from acute STEMI to 4 months' follow up (Table 2). Based on these data, we analysed sIL-1R2 further with respect to its association with markers of myocardial injury and LV remodelling.

\section{Soluble IL-1R2 is independently associated with measures of myocardial injury and remodelling after STEMI}

When the STEMI patients were dichotomized according to plasma levels of sIL-1R2 (i.e. above or below median) at different time points after STEMI, several significant findings were revealed. First, sIL-1R2 measured at day 1 were associated with infarct size $(p=0.04)$, $\operatorname{LVEF}(\mathrm{p}=0.03)$ and area at risk $(\mathrm{p}=0.003)$ during the acute phase (Table 3). Second, there was a significant association between presence of microvascular obstruction (MVO) and levels of sIL-1R2 measured immediately before and after PCI (Table 3; $\mathrm{p}=0.001$ and $\mathrm{p}=0.009$, respectively). Third, patients with high levels of sIL-1R2 before PCI also had lower LVEF determined by CMR at four months. Fourth, the change in LVEDVi and LVESVi from the acute phase to four months were significantly higher in patients with high (above median) compared to low (below median) levels of sIL1-R2 at admission (sampled immediately before and after PCI) and at day 1 (Figure 3A-B). Finally, sIL-1R2 at all sampling points during hospitalisation remained significantly associated with an increase in LVEDVi $\left(\approx 3.2-3.9 \mathrm{ml} / \mathrm{m}^{2}\right.$ per standard deviation change in sIL-1R2) and LVESVi $\left(\approx 2.4-3.0 \mathrm{ml} / \mathrm{m}^{2}\right.$ per standard deviation change in sIL-1R2) also after adjustment for relevant clinical covariates in two multivariable linear regression models (Figure 3C-D; Supplementary Table S5 and S6).

A similar approach with dichotomizing the patient group according to sIL-1RAcP levels, did not indicate any link between sIL-1RAcP and markers of LV remodelling, but 
there was a significant association between infarct size measured at 4 months and sIL-1RAcP measured after PCI (p=0.004) and at follow up ( $\mathrm{p}=0.008)$ (Supplementary Table S7).

\section{Discussion}

Dysregulated IL-1 signalling following MI can disturb infarct healing, but can also cause collateral damage and promote acute cardiac dysfunction and contribute to maladaptive LV remodelling. A complex regulation of IL-1 activity occurs at the receptor level, and here we show that levels of soluble regulators of IL-1 receptor signalling are changed in a population of patients with STEMI. Moreover, we show that plasma levels of sIL-1R2 remained elevated in the acute phase and during long-term follow-up, strongly associated with development of LV remodelling, as evaluated as change in LVEDVi and LVESVi, even after adjustment for multiple clinical covariates.

Levels of soluble IL-1 regulators in disease states have been reported in a few studies, including our previous study in patients with chronic HF [11-13]. Herein we examined these IL-1 regulators following STEMI. Notably, our findings on the temporal changes in IL-1Ra and sIL-1R2 in STEMI patients resemble results from patients with septic shock [14]. While plasma IL-1Ra peaked with high values during acute STEMI and decreased markedly up to day 1, sIL-1R2 levels were moderately higher than in healthy controls, but intriguingly, this difference was sustained even 12 months after the ischemic event. IL-1R2 is a decoy receptor with greater affinity for IL-1 $\beta$ than IL-1Ra [6], and these effects are maintained also when IL1R2 is shed from the cell surface. Conversely, IL-1Ra has higher affinity for IL-1R1 than for IL-1R2 and this avoids that the decoy receptor neutralizes the receptor antagonist. Thus, IL1Ra and sIL-1R2 represent two complementary mechanisms of negative IL-1 regulation, and during and after MI they have distinct temporal profiles. Previous studies have demonstrated 
an association between IL-1Ra and myocardial necrosis during MI $[8,15]$. We did, however, not observe this in the present study, but the correlation with CRP supports the previously reported association with the acute phase response and that IL-1Ra may be a marker of acute inflammation. In contrast, the increase in sIL-1R2 was more moderate and persisted throughout the observation period. Interestingly, whereas IL-1Ra may be a marker of acute systemic inflammation, the increase in sIL-1R2 has been suggested to be more targeted to local tissue inflammation [12]. Thus, it is tempting to hypothesize that sIL-1R2 is a better marker of IL-1 activity in the infarcted heart than IL-1Ra which is primarily a reflection of the acute phase response, but this will have to be more thoroughly addressed in future studies.

While most cells express IL-1R1, expression of IL-1R2 is restricted with neutrophils and monocytes as major sources [12]. Anti-inflammatory stimuli (e.g. glucocorticoids and IL4) increase neutrophil expression of IL-1R2, while inflammatory signals (e.g. IL-1, tumour necrosis factor $[\mathrm{TNF}]$ and reactive oxygen species) promote the proteolytic cleavage and release of sIL-1R2 [12]. Neutrophils are centrally involved in initiating inflammatory processes, but are also essential in inflammation resolution, and this at least partly involves expression and release of IL-1R2 [16, 17], which may dampen the response to IL-1 in both macrophages and fibroblasts $[18,19]$. Saxena et al. characterized the IL-1R expression profile in cells infiltrating the infarcted mouse myocardium [20] and demonstrated that the initial inflammatory phase of cardiac repair was characterized by cells primarily expressing IL-1R1, while IL-1R2 dominated in later stages [20]. This pattern fits well with what we observe during STEMI, with initially high levels of sIL-1R1 that decrease rapidly, while sIL-1R2 levels remain elevated. We hypothesize that even after the acute stages of STEMI, there is a persistent non-resolving IL-1 mediated myocardial inflammation, at least partly kept under control by IL-1R2, as reflected by elevated plasma levels of sIL-1R2. 
Inflammation has been suggested to be a significant contributor to ventricular remodelling after MI $[2,15,21]$. The major finding in the present study was that high levels of sIL-1R2 during the acute phase were significantly associated with ventricular remodelling evaluated as change in LVEDVi and LVESVi from the hospitalization to 4 months. An explanation for the association between high sIL-1R2 levels and adverse myocardial remodelling could be that increased sIL-1R2 levels represent a counteracting mechanism to enhanced inflammation such as has been reported in septicaemia [14]. This intriguing finding may also indicate that inhibiting IL-1 signalling by this soluble receptor, that strongly and irreversibly bind IL-1, may be harmful during the acute phase. Whereas IL-1Ra has been suggested to have beneficial effects when administered during MI [3], the near irreversible binding of sIL-1R2 to IL-1 during the acute phase could potentially attenuate adaptive remodelling and infarct healing induced by IL-1 and thereby promote increased infarct size and maladaptive remodelling during follow-up. This concept could have important therapeutical consequences when targeting the IL-1 system during MI.

Soluble IL-1RAcP appeared as the only IL-1 signalling regulator that was downregulated in the acute phase of STEMI. Soluble IL-1RAcP levels during the acute phase were also negatively correlated with infarct size both during the acute phase and at 4 months' follow-up as assessed by CMR imaging. IL-1RAcP is the main signalling unit of the IL-1 receptor complex [6]. However, when IL-1RAcP is solubilized, it turns into a negative regulator of IL-1 signalling [6]. This can potentially occur through inhibition of the interaction between IL-1R1/IL-1 and IL-1RAcP. However, sIL-1RAcP has also been shown to 100 -fold increase the affinity of IL-1 bound to sIL-1R2 [22], and interestingly, sIL-1R2 and sIL-1RAcP bind pro-IL-1 $\beta$ with high affinity and block its processing by caspase- 1 and thereby not only attenuate the effect of IL-1 $\beta$, but also its production [23]. The observed reduction of sIL-1RAcP could therefore have potentially pro-inflammatory net effects. 
However, its interaction with sIL-1R2 is complex and the net effect of the demonstrated pattern with increased sIL-1R2 and decreased sIL-1RAcP is at present not clear.

The present study has some limitations such as lack of exact data on lipid levels on the STEMI patients prior to admission (i.e., before start of statin therapy). Moreover, levels of IL$1 \mathrm{Ra}$ have previously been found to be elevated in patients with stable coronary artery disease [24], but we do not know if levels the other soluble IL-1 regulators are affected by the atherosclerotic process per se. Future studies will have to address this issue, which could potentially affect how we interpret the effect of STEMI on levels of soluble levels of IL-1 signalling. Nonetheless, our data demonstrate a complex regulation of the IL-1 system during STEMI, and suggest that sIL-1R2 could be a hereto unrecognized mediator and marker of myocardial remodelling in these patients. Our data also illustrate the delicate balance between too much and too little IL-1 activity following MI.

\section{Funding}

This work was supported by grants from Helse Sør-Øst Regional Health Authority, Norway [grant number 2012037 to A.Y.]; Norwegian Research Council [grant number 240099/F20 to P.A.]; and the European Community's Seventh Framework Programme [grant number 602699 to T.E.M.]. The study was also supported by The Norwegian Council on Cardiovascular Disease, The Odd Fellow Foundation and The Simon Fougner Hartmann Family Fund. Funding sources had no involvement in study design, analysis/study design, or in writing of the manuscript.

\section{Conflict of Interest}

None declared. 


\section{References}

1. Frangogiannis NG. The inflammatory response in myocardial injury, repair, and remodelling. Nat Rev Cardiol 2014;11:255-65.

2. Westman PC, Lipinski MJ, Luger D et al. Inflammation as a Driver of Adverse Left Ventricular Remodeling After Acute Myocardial Infarction. J Am Coll Cardiol 2016;67:205060.

3. Van Tassell BW, Toldo S, Mezzaroma E, Abbate A. Targeting interleukin-1 in heart disease. Circulation 2013;128:1910-23.

4. Morton AC, Rothman AM, Greenwood JP et al. The effect of interleukin-1 receptor antagonist therapy on markers of inflammation in non-ST elevation acute coronary syndromes: the MRC-ILA Heart Study. Eur Heart J 2015;36:377-84.

5. Ridker PM, Everett BM, Thuren T et al. Antiinflammatory Therapy with Canakinumab for Atherosclerotic Disease. N Engl J Med 2017;377:1119-31.

6. Gabay C, Lamacchia C, Palmer G. IL-1 pathways in inflammation and human diseases. Nat Rev Rheumatol 2010;6:232-41.

7. Orn S, Ueland T, Manhenke C et al. Increased interleukin-1beta levels are associated with left ventricular hypertrophy and remodelling following acute ST segment elevation myocardial infarction treated by primary percutaneous coronary intervention. J Intern Med 2012;272:267-76.

8. Patti G, D'Ambrosio A, Mega S et al. Early interleukin-1 receptor antagonist elevation in patients with acute myocardial infarction. J Am Coll Cardiol 2004;43:35-8.

9. Debrunner M, Schuiki E, Minder E et al. Proinflammatory cytokines in acute myocardial infarction with and without cardiogenic shock. Clin Res Cardiol 2008;97:298-305. 
10. Limalanathan S, Andersen GO, Klow NE, Abdelnoor M, Hoffmann P, Eritsland J. Effect of ischemic postconditioning on infarct size in patients with ST-elevation myocardial infarction treated by primary PCI results of the POSTEMI (POstconditioning in ST-Elevation Myocardial Infarction) randomized trial. J Am Heart Assoc 2014;3:e00679.

11. Garlanda C, Riva F, Bonavita E, Mantovani A. Negative regulatory receptors of the IL-1 family. Semin Immunol 2013;25:408-15.

12. Peters VA, Joesting JJ, Freund GG. IL-1 receptor 2 (IL-1R2) and its role in immune regulation. Brain Behav Immun 2013;32:1-8.

13. Aukrust $\mathrm{P}$, Ueland T, Lien E et al. Cytokine network in congestive heart failure secondary to ischemic or idiopathic dilated cardiomyopathy. Am J Cardiol 1999;83:376-82. 14. van Deuren M, van der Ven-Jongekrijg J, Vannier E et al. The pattern of interleukin1beta (IL-1beta) and its modulating agents IL-1 receptor antagonist and IL-1 soluble receptor type II in acute meningococcal infections. Blood 1997;90:1101-8.

15. Seropian IM, Sonnino C, Van Tassell BW, Biasucci LM, Abbate A. Inflammatory markers in ST-elevation acute myocardial infarction. Eur Heart J Acute Cardiovasc Care 2016;5:382-95.

16. Bourke E, Cassetti A, Villa A, Fadlon E, Colotta F, Mantovani A. IL-1 beta scavenging by the type II IL-1 decoy receptor in human neutrophils. J Immunol 2003;170:5999-6005.

17. Mantovani A, Cassatella MA, Costantini C, Jaillon S. Neutrophils in the activation and regulation of innate and adaptive immunity. Nat Rev Immunol 2011;11:519-31.

18. Shimizu K, Nakajima A, Sudo K et al. IL-1 receptor type 2 suppresses collageninduced arthritis by inhibiting IL-1 signal on macrophages. J Immunol 2015;194:3156-68. 
19. Martin P, Palmer G, Rodriguez E et al. Deficiency in IL-1 Receptor Type 2

Aggravates K/BxN Serum Transfer-Induced Arthritis in Mice but Has No Impact on Systemic Inflammatory Responses. J Immunol 2017;198:2916-26.

20. Saxena A, Chen W, Su Y et al. IL-1 induces proinflammatory leukocyte infiltration and regulates fibroblast phenotype in the infarcted myocardium. J Immunol 2013;191:4838-48. 21. Seropian IM, Toldo S, Van Tassell BW, Abbate A. Anti-inflammatory strategies for ventricular remodeling following ST-segment elevation acute myocardial infarction. $J$ Am Coll Cardiol 2014;63:1593-603.

22. Smith DE, Hanna R, Della F et al. The soluble form of IL-1 receptor accessory protein enhances the ability of soluble type II IL-1 receptor to inhibit IL-1 action. Immunity 2003;18:87-96.

23. Symons JA, Young PR, Duff GW. Soluble type II interleukin 1 (IL-1) receptor binds and blocks processing of IL-1 beta precursor and loses affinity for IL-1 receptor antagonist. Proc Natl Acad Sci U S A 1995;92:1714-8.

24. Gabriel AS, Ahnve S, Wretlind B, Martinsson A. IL-6 and IL-1 receptor antagonist in stable angina pectoris and relation of IL-6 to clinical findings in acute myocardial infarction. $J$ Intern Med 2000;248:61-6.

Corresponding author:

Arne Yndestad, Research Institute of Internal Medicine, Oslo University Hospital, Oslo

Email: arne.yndestad@medisin.uio.no ,Tel:+47-41217144, Fax:+47-23073630.

Alternative proof reader:

Hilde L. Orrem, Department of Anesthesiology, Oslo University Hospital Rikshospitalet, Oslo. Email: hilde.orrem@gmail.com. 


\section{Figure legends}

\section{Figure 1. Regulators of Interleukin (IL)-1 signalling.}

IL-1 (IL-1 $\alpha$ or IL-1 $\beta$ ) binds to IL-1 receptor 1 (IL-1R1), IL-1R accessory protein (IL-1RAcP) is recruited and signaling initiated (A). IL-1 receptor antagonist (IL-1Ra) binds IL-1R1 with high specificity, but does not trigger downstream signaling (B). IL-1R2 binds IL-1, but do not initiate signaling (C). Soluble (s) IL-1R2 binds and neutralizes IL-1 with high affinity (D), while sIL-1R1 has preferential affinity for IL-1Ra (E). Soluble (s) IL-1RAcP may either inhibit formation of the signaling complex (F) or stabilize the binding of IL-1 to sIL-1R2 (G).

Figure 2. Temporal profile of soluble regulators of IL-1 signalling during the course of STEMI.

Levels of (A) IL-1 receptor antagonist (IL-1Ra), (B) soluble IL-1 receptor 1 (sIL-1R1), (C) sIL-1R2 and (D) sIL-1R Accessory protein (sIL-1RAcP) were measured in 65 healthy controls and 255 STEMI patients before and immediately after percutaneous coronary intervention (PCI), at Day 1 (median 18.3 hours after PCI) and at 4 and 12 months' follow-up. Circles and error bars indicate geometric means and $95 \%$ confidence intervals. $* * \mathrm{p}<0.01$, $* * * \mathrm{p}<0.001$ and $* * * * \mathrm{p}<0.0001$ vs controls (Kruskal-Wallis comparing controls and STEMI at different time points with Dunn's multiple comparisons test). P-values in graphs indicate results of univariate repeated measures ANOVA on temporal profile in STEMI patients. 


\section{Figure 3. Soluble interleukin-1 receptor 2 (sIL-1R2) levels are associated with}

ventricular remodelling after STEMI.

sIL-1R2 levels were measured before and immediately after PCI and at Day 1 (median 18.3 hours after PCI). Change in indexed left ventricular end-diastolic volume (LVEDVi) and endsystolic volume (LVESVi) were determined in 224 patients with ST-elevation myocardial infarction (STEMI) based on CMR in the acute phase and at 4-months' follow-up. (A). Change in LVEDVi and (B) change in LVESVi according to low or high levels of sIL-1R2 (i.e. $<$ or $>$ median) in the acute phase of STEMI. Bars are means $\pm 95 \%$ confidence intervals (CI). C and D. Forest plots of $\beta$ coefficients, $95 \%$ CI and p-values from univariable (Uni) and multivariable linear regression analyses on associations between sIL-1R2 measured at day 1 post-STEMI and changes in LVEDVi (C) and LVESVi (D). Model 1 adjusts for clinical variables, while Model 2 also includes C-reactive protein (CRP) and troponin T (TnT). Results from the most important adjustment variables are shown for comparison. Coefficients $(\beta)$ are expressed as change in LVEDVi or LVESVi per 1 standard deviation (SD) change in the independent variable. Adm, admission; NT-proBNP, N-terminal pro-brain natriuretic peptide. $* * \mathrm{p}<0.01$ and $* * * \mathrm{p}<0.001 \mathrm{vs}<$ median (t-test). 
Table 1. Baseline characteristics of the patient population in the POSTEMI trial.

\section{Patients with ST-elevation myocardial infarction}

$$
(n=272)
$$

$\begin{array}{lc}\text { Age } & 60(53,67) \\ \text { Male gender } & 223(82 \%) \\ \text { Body mass index }\left(\mathrm{kg} / \mathrm{m}^{2}\right) & 26.6(24.4,29.1) \\ \text { Hypertension } & 73(26.8 \%) \\ \text { Hypercholesterolemia } & 26(9.6 \%) \\ \text { Diabetes mellitus } & 17(6.3 \%) \\ \text { Current smoker } & 139(51.1 \%) \\ \text { Time from symptom to PCI (min) } & 179(123,261) \\ \text { Anterior MI* } & 131(48.2 \%) \\ \text { Multi-vessel disease } & 90(33.1 \%) \\ \text { Peak troponin T (ng/L) } & 5865(3302,10337) \\ \text { Peak C-reactive protein }(\mathrm{mg} / \mathrm{L}) & 18(7,45) \\ \text { Admission NT-proBNP }(\mathrm{pmol} / \mathrm{L}) & 9(5,22) \\ \text { Admission creatinine }(\mu \mathrm{mol} / \mathrm{L}) & 70(62,81) \\ \end{array}$

Data are presented as median (25th, 75th percentile) or numbers $(\%) . *$ Anterior myocardial infarction (MI) vs inferior or posterior MI. POSTEMI, postconditioning in ST-elevation myocardial infarction; PCI, percutaneous coronary intervention; NT-proBNP, N-terminal probrain natriuretic peptide. 
Table 2. Correlations between soluble IL-1 regulators and measures of cardiac injury and function.

\begin{tabular}{lcccc}
\hline & IL-1Ra & sIL-1R1 & sIL-1R2 & sIL-1RAcP \\
\hline Infarct size, acute & 0.06 & 0.000 & $\mathbf{0 . 1 5}$ & $\mathbf{- 0 . 1 9 * *}$ \\
Infarct size, 4 months & 0.08 & -0.02 & 0.09 & $\mathbf{- 0 . 1 7 *}$ \\
Ejection fraction, acute & -0.14 & -0.07 & $\mathbf{- 0 . 1 5 *}$ & 0.06 \\
Ejection fraction, 4 months & -0.07 & -0.06 & -0.12 & 0.10 \\
Area at risk & 0.07 & -0.07 & 0.12 & -0.11 \\
Myocardial salvage & -0.03 & -0.06 & -0.10 & 0.09 \\
$\Delta$ LVEDVi & 0.10 & -0.02 & $\mathbf{0 . 2 1 * *}$ & -0.04 \\
$\Delta$ LVESVi & 0.12 & -0.05 & $\mathbf{0 . 1 6 *}$ & -0.06 \\
Peak troponin T & 0.10 & 0.01 & $\mathbf{- 0 . 1 7 * *}$ & $\mathbf{- 0 . 1 7 * *}$ \\
Peak C-reactive protein & $\mathbf{0 . 3 5 * *}$ & 0.03 & $\mathbf{0 . 1 9 * *}$ & -0.13 \\
Admission NT-proBNP & 0.09 & 0.12 & $\mathbf{- 0 . 2 0 * *}$ & -0.03 \\
Symptom to PCI time & -0.03 & -0.11 & -0.06 & $\mathbf{- 0 . 1 4 *}$ \\
\hline Correlations an & & & & \\
\hline
\end{tabular}

Correlations are based on plasma levels of interleukin-1 receptor antagonist (IL-1Ra), soluble (s) IL-1R1, sIL-1R2 and sIL-1R accessory protein (sIL-1RAcP) measured day 1 (median 18.3 hours after PCI) after ST-elevation myocardial infarction (STEMI; n=255-272). $\triangle$ LVEDVi (indexed end-diastolic volume of LV) and $\triangle$ LVESVi (indexed end-systolic volume of LV) were calculated from cardiac magnetic resonance imaging acquired in the acute phase and after 4 months. NT-proBNP, N-terminal pro-brain natriuretic peptide. Numbers are given as Spearman's rho correlation coefficients. ${ }^{*} \mathrm{p}<0.05,{ }^{* *} \mathrm{p}<0.01$. 
Table 3. Myocardial injury and function measured by CMR according to sIL-1R2 values.

\begin{tabular}{|c|c|c|c|c|c|c|c|c|c|c|c|c|}
\hline & \multicolumn{3}{|c|}{ sIL-1R2 before PCI } & \multicolumn{3}{|c|}{ sIL-1R2 after PCI } & \multicolumn{3}{|c|}{ sIL-1R2 day 1} & \multicolumn{3}{|c|}{ sIL-1R2 4 months } \\
\hline & $\leq$ median & $>$ median & P-value & $\leq$ median & $>$ median & P-value & $\leq$ median & $>$ median & P-value & $\leq$ median & $>$ median & P-value \\
\hline \multicolumn{13}{|l|}{ CMR in } \\
\hline \multicolumn{13}{|l|}{ acute phase } \\
\hline IS, \%LV mass & 15.8 & 18.2 & 0.07 & 16.6 & 17.9 & 0.45 & 15.5 & 19.2 & 0.04 & & & \\
\hline LVEF, \% & 52 & 51 & 0.30 & 52 & 51 & 0.40 & 53 & 49 & 0.03 & & & \\
\hline Area at risk, \%LV & 41.1 & 43.5 & 0.15 & 40.8 & 43.3 & 0.11 & 38.0 & 44.8 & 0.003 & & & \\
\hline MVO (presence) & $41(39.4 \%)$ & $68(61.3 \%)$ & 0.001 & $46(41.1 \%)$ & $64(58.7 \%)$ & 0.009 & $47(44.8 \%)$ & $57(53.8 \%)$ & 0.19 & & & \\
\hline LVEDVi, mL/m² & 83.7 & 83.8 & 0.97 & 84.8 & 80.3 & 0.31 & 83.6 & 84.2 & 0.88 & & & \\
\hline LVESVi, mL/m² & 38.8 & 39.9 & 0.66 & 41.2 & 38.7 & 0.97 & 38.2 & 41.5 & 0.22 & & & \\
\hline \multicolumn{13}{|l|}{ CMR after } \\
\hline \multicolumn{13}{|l|}{4 months } \\
\hline IS, \%LV mass & 13.6 & 14.5 & 0.13 & 13.8 & 13.8 & 0.33 & 13.5 & 13.8 & 0.28 & 14.0 & 14.0 & 0.44 \\
\hline LVEF, \% & 58 & 53 & 0.006 & 57 & 54 & 0.11 & 57 & 55 & 0.13 & 56 & 55 & 0.57 \\
\hline MS, $\%$ & 54.4 & 48.4 & 0.07 & 54.2 & 50.5 & 0.07 & 51.0 & 52.2 & 0.75 & 52.5 & 51.1 & 0.18 \\
\hline LVEDVi, mL/m² & 80.5 & 88.6 & 0.006 & 85.3 & 86.7 & 0.19 & 84.8 & 86.7 & 0.09 & 86.1 & 86.8 & 0.61 \\
\hline LVESVi, $\mathrm{mL} / \mathrm{m}^{2}$ & 33.3 & 39.8 & 0.002 & 36.4 & 38.0 & 0.11 & 35.9 & 38.0 & 0.08 & 37.9 & 37.9 & 0.54 \\
\hline
\end{tabular}

Levels of soluble interleukin-1 receptor 2 (sIL-1R2) were measured before and immediately after percutaneous coronary intervention (PCI), at Day 1 (median 18.3 hours after PCI) and at 4-months' follow-up in 255 ST-elevation myocardial infarction (STEMI) patients. Data are presented as median or numbers (\%). P-values were determined by Mann-Whitney U test. CMR, cardiac magnetic resonance imaging; IS, infarct size; LV, left ventricle; LVEF, LV ejection fraction; MVO, microvascular obstruction; LVEDVi, indexed end-diastolic volume of LV; LVESVi, indexed end-systolic volume of LV; MS, myocardial salvage. 
Figure 1

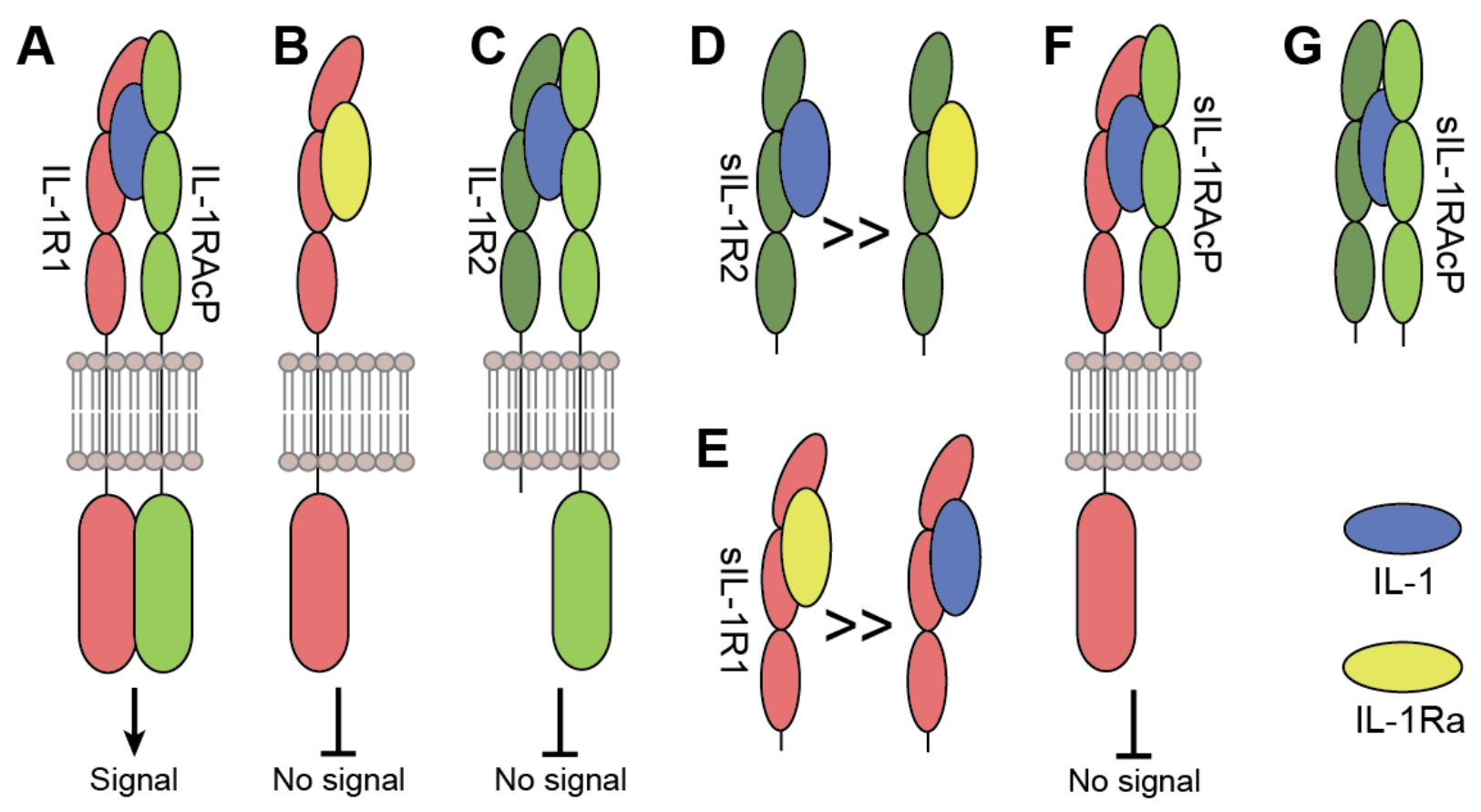


Figure 2
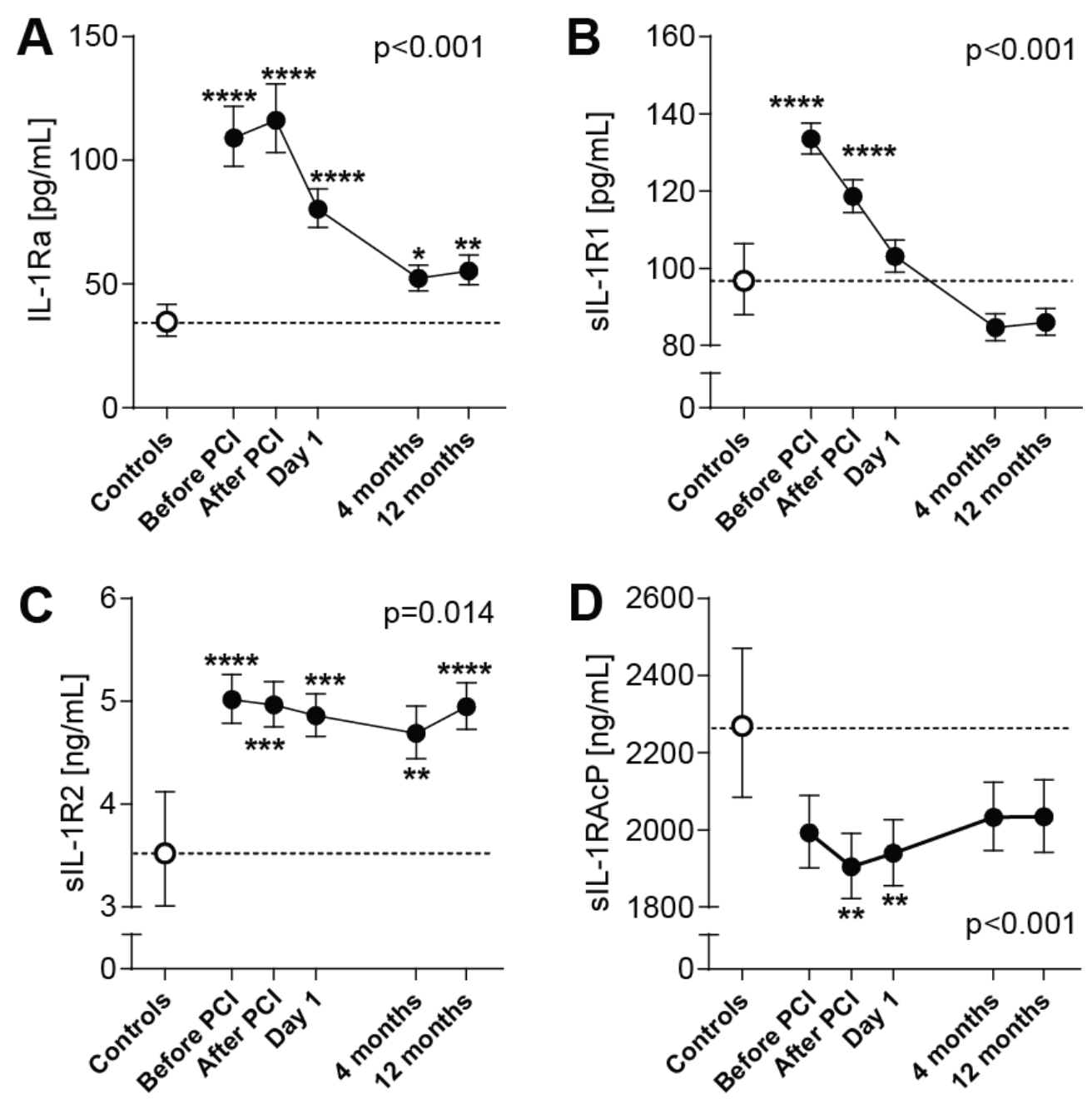


\section{Figure 3}
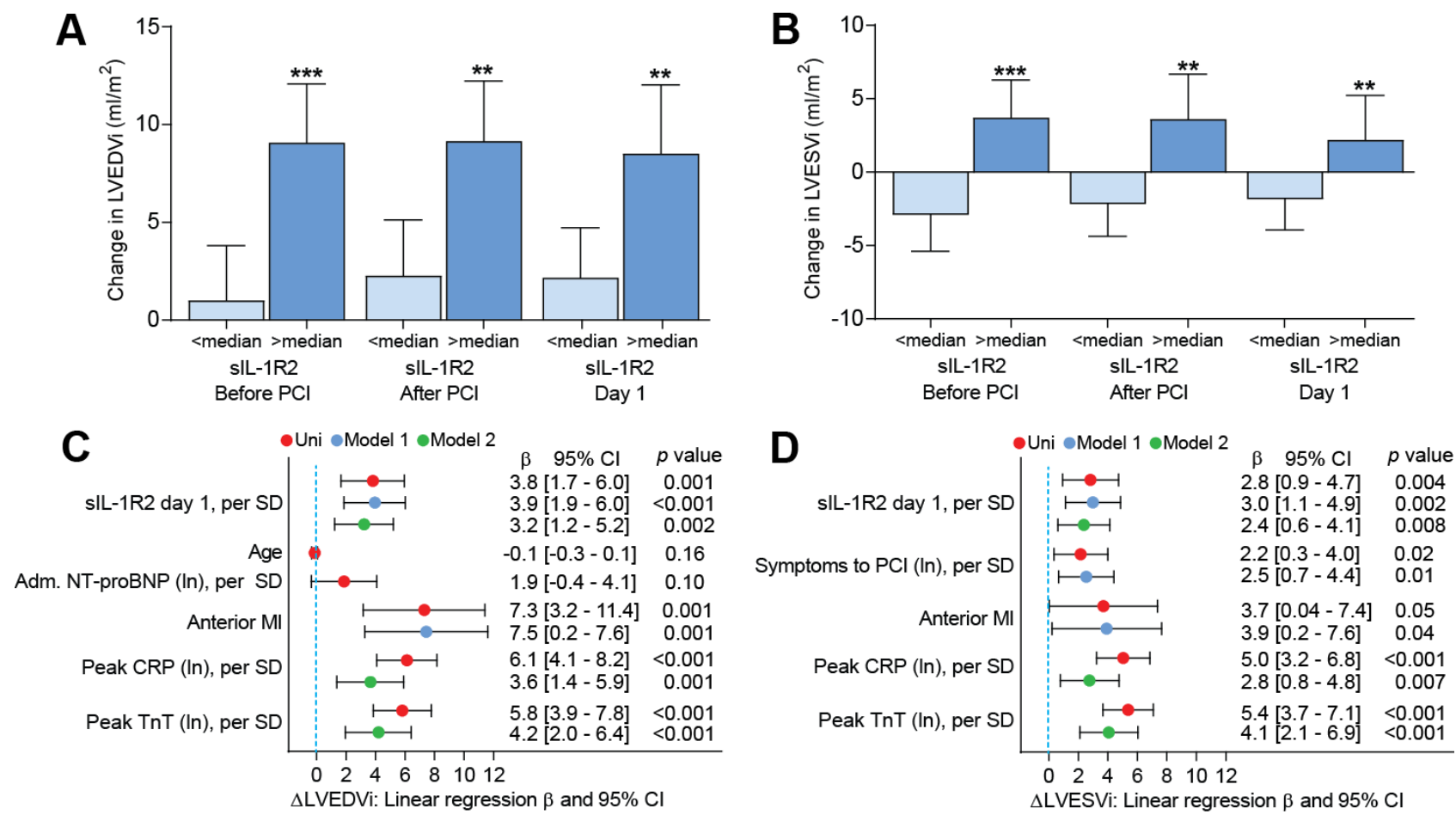